\title{
Nothing new under the nuclear sun: towards 80 years of theranostics in nuclear medicine
}

\author{
Frederik A. Verburg • Alexander Heinzel • \\ Heribert Hänscheid • Felix M. Mottaghy • \\ Markus Luster • Luca Giovanella
}

Published online: 6 November 2013

(C) Springer-Verlag Berlin Heidelberg 2013

Some time in the early 2000s, the word "theranostics" (or "theragnostics") started surfacing in the medical literature. Theranostics (from the Greek therapeuein "to treat medically" and gnosis "knowledge") is the use of individual patient-level biological information in choosing the optimal therapy for that individual [1]. In the modern era of "personalized medicine", theranostics is increasingly pursued in many branches of medicine in order to develop ever more effective treatment regimens. There are now many studies and reviews dedicated to theranostics, and even a journal bearing the name of this principle, detailing many different concepts on how to combine imaging and therapy using, for example, complex molecules [2] or nanotechnology [3].

However, it is rarely realized by either clinicians or scientists that nuclear medicine has been employing theranostics for nearly 80 years now. In fact, the very foundations of targeted therapy in nuclear medicine are those that are only now being adopted by other medical disciplines under the designation "theranostics".

F. A. Verburg $(\bowtie) \cdot$ A. Heinzel $\cdot$ F. M. Mottaghy

Department of Nuclear Medicine, RWTH University Hospital

Aachen, Pauwelsstraße 30, 52074 Aachen, Germany

e-mail: fverburg@ukaachen.de

\section{F. A. Verburg · F. M. Mottaghy}

Department of Nuclear Medicine, Maastricht University Medical

Center, Maastricht, The Netherlands

H. Hänscheid

Department of Nuclear Medicine, University of Wuerzburg,

Wuerzburg, Germany

\section{Luster}

Department of Nuclear Medicine, University Hospitals

Giessen-Marburg, Marburg, Germany

L. Giovanella

Department of Nuclear Medicine, Oncology Institute of Southern

Switzerland, Bellinzona, Switzerland
The cornerstones of theranostics can be traced back to some of the most illustrious names among the founding fathers of nuclear medicine. Soon after Chiewitz and de Hevesy [4] described the uptake of radioactive ${ }^{32} \mathrm{P}$ in the bones of rats, Erf and J.H. Lawrence (brother of the physicist Ernest O. Lawrence, who built the first cyclotron) applied this same radioisotope to patients suffering from leukaemia and polycythaemia vera [5]. Although this treatment certainly was not without success, it has since been superseded by more effective nonradioactive chemotherapy. Shortly afterwards Pecher [6] discovered that ${ }^{89} \mathrm{Sr}$ accumulated in secondary bone tumours in animals, and subsequently successfully used this radioisotope to treat patients with painful bone metastases (unfortunately this work was immediately classified as secret and it took more than five decades for ${ }^{89} \mathrm{Sr}$ to be registered as a therapeutic drug). These two studies are perhaps the earliest examples of diagnostic studies leading to targeted therapy of cancer using radionuclides.

Around the same time the most prominent example of pure nuclear theranostic medicine emerged: the diagnosis and treatment of thyroid disorders using various isotopes of iodine. Hertz et al. in 1938 described the first study of thyroidal radioiodine uptake [7], and in 1942 Hertz and Roberts reported on the treatment of the first patients with Graves' disease with radioiodine [8]. A short time later Seidlin et al. treated the first patient with metastatic thyroid cancer with radioiodine [9] - at the time this compound was so rare that radioiodine was purified from the patient's urine and readministered. During this therapy, additional metastases were identified using a Geiger counter and the first rudimentary dosimetry was performed. It is of course only with the benefit of hindsight that we can now say that this was the first application of theranostics in targeted molecular medicine through a specific molecular target, the sodium iodine symporter, long before any of these concepts were first described as "theranostics". Indeed, even today it is hard to think of a single combination of targeted diagnostics and therapy that is more specific than radioiodine. 
Since then radioactive iodine has been clearly established for pretherapeutic dosimetry [10] and for the therapy of benign and malignant thyroid disease, as well as for posttherapeutic diagnostic imaging during follow-up in the form of diagnostic whole-body scintigraphy. It is likely that in the future the use of radioactive iodine for treatment of thyroid diseases can and will have to be personalized further in order to optimize the chance of therapeutic success [11], possibly in combination with other therapeutic agents [12]. Another proven approach is the labelling of other substances with ${ }^{131} \mathrm{I}$. Metaiodobenzylguanidine (MIBG) [13] in the diagnosis and treatment of neuroblastoma, phaeochromocytoma and paragangliomas and the $11-\beta$-hydroxylase substrate ${ }^{131} \mathrm{I}-$ iodometomidate for targeted diagnosis and radionuclide therapy of advanced adrenocortical carcinoma make use of the same theranostic principles [14].

A further excellent example of theranostics in nuclear medicine, that was introduced much more recently, concerns the diagnostic and therapeutic targeting of the somatostatin receptor. First developed in the late 1980s, the original concept was to perform both imaging and therapy in patients with neuroendocrine tumours using ${ }^{111}$ In-labelled octreotide (a somatostatin analogue) [15]. Although ${ }^{111}$ In-octreotide was eventually successfully registered and marketed as a diagnostic radiopharmaceutical, new somatostatin analogues such as [DOTA0,Tyr3]-octreotide (DOTATOC) and [DOTA0, Tyr3]-octreotate (DOTATATE) labelled with ${ }^{177} \mathrm{Lu}$ or ${ }^{90} \mathrm{Y}[16$, 17] were more successful alternatives for peptide receptor radiotherapy (PRRT) in patients with tumours positive for somatostatin receptor had been diagnosed with ${ }^{111} \mathrm{In}$ octreotide. Personalization of PRRT, for example to ensure that the critical dose to the kidneys is not exceeded, can be performed using dosimetry with ${ }^{111}$ In-octreotide or ${ }^{86} \mathrm{Y}$ labelled DOTATOC/DOTATATE [18]. Posttherapy dosimetric monitoring has also been reported extensively [19].

More recently new indications for PRRT, such as for the treatment of meningeomas, have emerged [20]. In this indication, it was found that the delivered dose of PRRT of meningeomas could be predicted even when the somatostatin analogue was labelled with the short-lived positron emitter ${ }^{68} \mathrm{Ga}$ [19], opening up the possibility of better selection of patients for PRRT on the basis of quantitative analysis of a single diagnostic DOTATOC/DOTATATE PET/CT scan.

Aside from using nuclear medicine methods in the selection for and monitoring of radionuclide therapies in patients, the principle of nuclear theranostics is arriving in oncology as well. In recent years, a ${ }^{99 \mathrm{~m}} \mathrm{Tc}$-labelled chemotherapy analogue was developed which can be used for the selection of patients with tumours expressing folate receptors, enabling the selection of those patients who will benefit from chemotherapy with folate receptor-targeted agents while sparing those patients who do not express this receptor a potentially toxic but probably ineffective course of treatment [21].
The concept of theranostics in nuclear medicine can even be expanded well beyond the specific transporter or receptor based diagnostics and therapies. In the broader sense, the use of ${ }^{18} \mathrm{~F}$-fluorodeoxyglucose (FDG) PET/CT in oncology is a form of theranostics, especially when linked to a decision on therapy. This is especially evident in, for example, modern protocols for the treatment of lymphoma, where the decision on additional treatment with, for example, external beam radiotherapy (EBRT) depends on whether FDG-positive lesions are still present after a number of chemotherapy cycles [22]. In modern EBRT, PET/CT using various tracers such as FDG or ${ }^{18} \mathrm{~F}$-fluoroethyltyrosine is also a clear diagnostic test which is linked to a specific therapy, as modern radiotherapy approaches use PET/CT for planning the extent of EBRT, as well as to determine where an additional radiation boost needs to be applied in intensity-modulated radiotherapy [23].

In addition, a third aspect of nuclear medicine that is hidden that is in the word "theranostics" as much as therapeutics and diagnostics is prognostics. Many diagnostic tests in nuclear medicine are associated with a clear prognostic stratification. For instance, after ${ }^{131} \mathrm{I}$ ablation a negative first diagnostic whole-body ${ }^{131}$ I scintigraphy in combination with a negative thyroglobulin level and a negative ultrasound scan of the neck not only indicates that no further ${ }^{131} \mathrm{I}$ therapy is necessary but is also clearly associated with a lower risk of tumour recurrence and thyroid cancer-related death [24] - especially in pretherapeutically high-risk patients, in whom the risk of recurrence or death from thyroid cancer is reduced to that in pretherapeutically low-risk patients [25]. In another example, FDG PET/CT for many indications has clearly been shown to be associated with prognosis, either through uptake intensity or through metabolic volume, in a range of cancers encompassing nearly the entire spectrum of medical oncology, including primary examples such as lung cancer [26], breast cancer [27], oesophageal cancer [28] and lymphoma [29]. This may also hold true for other PET/CT tracers such as FET [30].

The above is just a small selection of an ever-growing number [31] of examples which clearly illustrate that nuclear medicine has had both a rich history and an exemplary role in establishing the basics of the theranostic concept as well as in the development of personalized medicine in general. We as nuclear medicine physicians should recognize and use this to gain a central role in the future development of new theranostic approaches in personalized medicine.

\section{References}

1. Bentzen SM. Theragnostic imaging for radiation oncology: dosepainting by numbers. Lancet Oncol. 2005;6:112-7.

2. Zhu G, Zheng J, Song E, Donovan M, Zhang K, Liu C, et al. Selfassembled, aptamer-tethered DNA nanotrains for targeted transport of molecular drugs in cancer theranostics. Proc Natl Acad Sci U S A. 2013;110:7998-8003. 
3. Chen J, Shao R, Zhang XD, Chen C. Applications of nanotechnology for melanoma treatment, diagnosis, and theranostics. Int $\mathrm{J}$ Nanomedicine. 2013;8:2677-88.

4. Chiewitz O, de Hevesy G. Radioactive indicators in the study of phosphorus metabolism in rats. Nature. 1935;136:754-5.

5. Erf LA, Lawrence JH. Clinical studies with the aid of radioactive phosphorus. I. The absorption and distribution of radio-phosphorus in the blood and its excretion by normal individuals and patients with leukemia. J Clin Invest. 1941;20:567-75.

6. Pecher P. Biological investigation with radioactive calcium and strontium. Preliminary report on the use of radioactive strontium in the treatment of metastatic bone cancer. Univ Calif Publ Pharmacol. 1942;2:149.

7. Hertz S, Roberts A, Evans RD. Radioactive iodine as an indicator in the study of thyroid physiology. Proc Soc Exp Biol Med. 1938;38: 510-3.

8. Hertz S, Roberts A. Application of radioactive iodine in Graves disease. J Clin Invest. 1942;21:624.

9. Seidlin SM, Marinelli LD, Oshry E. Radioactive iodine therapy: effect on functioning metastases of adenocarcinoma of the thyroid. JAMA. 1946;132:838-47.

10. Lassmann M, Haenscheid H, Chiesa C, Hindorf C, Flux G, Luster M. EANM dosimetry committee series on standard operational procedures for pre-therapeutic dosimetry I: blood and bone marrow dosimetry in differentiated thyroid cancer therapy. Eur J Nucl Med Mol Imaging. 2008;35:1405-12.

11. Verburg FA, Lassmann M, Mader U, Luster M, Reiners C, Hanscheid $\mathrm{H}$. The absorbed dose to the blood is a better predictor of ablation success than the administered 131 I activity in thyroid cancer patients. Eur J Nucl Med Mol Imaging. 2011;38:673-80.

12. Ho AL, Grewal RK, Leboeuf R, Sherman EJ, Pfister DG, Deandreis $\mathrm{D}$, et al. Selumetinib-enhanced radioiodine uptake in advanced thyroid cancer. N Engl J Med. 2013;368:623-32.

13. Sisson JC, Yanik GA. Theranostics: evolution of the radiopharmaceutical meta-iodobenzylguanidine in endocrine tumors. Semin Nucl Med. 2012;42:171-84.

14. Hahner S, Kreissl MC, Fassnacht M, Haenscheid H, Bock S, Verburg FA, et al. Functional characterization of adrenal lesions using [123I]IMTO-SPECT/CT. J Clin Endocrinol Metab. 2013;98:1508-18.

15. Krenning EP, Kooij PP, Bakker WH, Breeman WA, Postema PT, Kwekkeboom DJ, et al. Radiotherapy with a radiolabeled somatostatin analogue, [111In-DTPA-D-Phe1]-octreotide. A case history. Ann N Y Acad Sci. 1994;733:496-506.

16. De Jong M, Breeman WA, Bernard BF, Bakker WH, Schaar M, van Gameren A, et al. [177Lu-DOTA(0),Tyr3] octreotate for somatostatin receptor-targeted radionuclide therapy. Int J Cancer. 2001;92:628-33.

17. De Jong M, Bakker WH, Krenning EP, Breeman WA, van der Pluijm ME, Bernard BF, et al. Yttrium-90 and indium-111 labelling, receptor binding and biodistribution of [DOTA0, d-Phe1, Tyr3]octreotide, a promising somatostatin analogue for radionuclide therapy. Eur J Nucl Med. 1997;24:368-71.

18. Helisch A, Forster GJ, Reber H, Buchholz HG, Arnold R, Göke B, et al. Pre-therapeutic dosimetry and biodistribution of 86Y-DOTA-
Phe1-Tyr3-octreotide versus 111In-pentetreotide in patients with advanced neuroendocrine tumours. Eur J Nucl Med Mol Imaging. 2004;31:1386-92.

19. Hanscheid H, Sweeney RA, Flentje M, Buck AK, Löhr M, Samnick $\mathrm{S}$, et al. PET SUV correlates with radionuclide uptake in peptide receptor therapy in meningioma. Eur J Nucl Med Mol Imaging. 2012;39:1284-8.

20. Kreissl MC, Hanscheid H, Lohr M, Verburg FA, Schiller M, Lassmann $\mathrm{M}$, et al. Combination of peptide receptor radionuclide therapy with fractionated external beam radiotherapy for treatment of advanced symptomatic meningioma. Radiat Oncol. 2012;7:99.

21. Edelman MJ, Harb WA, Pal SE, Boccia RV, Kraut MJ, Bonomi P, et al. Multicenter trial of EC145 in advanced, folate-receptor positive adenocarcinoma of the lung. J Thorac Oncol. 2012;7:1618-21.

22. Engert A, Haverkamp H, Kobe C, Markova J, Renner C, Ho A, et al. Reduced-intensity chemotherapy and PET-guided radiotherapy in patients with advanced stage Hodgkin's lymphoma (HD15 trial): a randomised, open-label, phase 3 non-inferiority trial. Lancet. 2012;379:1791-9.

23. Piroth MD, Pinkawa M, Holy R, Klotz J, Schaar S, Stoffels G, et al. Integrated boost IMRT with FET-PET-adapted local dose escalation in glioblastomas. Results of a prospective phase II study. Strahlenther Onkol. 2012;188:334-9.

24. Verburg FA, de Keizer B, Lips CJ, Zelissen PM, de Klerk JM. Prognostic significance of successful ablation with radioiodine of differentiated thyroid cancer patients. Eur J Endocrinol. 2005;152: 33-7.

25. Verburg FA, Stokkel MP, Duren C, Verkooijen RB, Mäder U, van Isselt JW, et al. No survival difference after successful (131)I ablation between patients with initially low-risk and high-risk differentiated thyroid cancer. Eur J Nucl Med Mol Imaging. 2010;37:276-83.

26. Liao S, Penney BC, Wroblewski K, Zhang H, Simon CA, Kampalath $\mathrm{R}$, et al. Prognostic value of metabolic tumor burden on 18F-FDG PET in nonsurgical patients with non-small cell lung cancer. Eur $\mathrm{J}$ Nucl Med Mol Imaging. 2012;39:27-38.

27. Evangelista L, Baretta Z, Vinante L, Cervino AR, Gregianin M, Ghiotto C, et al. Tumour markers and FDG PET/CT for prediction of disease relapse in patients with breast cancer. Eur J Nucl Med Mol Imaging. 2011;38:293-301.

28. Barber TW, Duong CP, Leong T, Bressel M, Drummond EG, Hicks RJ. 18F-FDG PET/CT has a high impact on patient management and provides powerful prognostic stratification in the primary staging of esophageal cancer: a prospective study with mature survival data. J Nucl Med. 2012;53:864-71.

29. Zinzani PL, Rigacci L, Stefoni V, Broccoli A, Puccini B, Castagnoli A, et al. Early interim 18F-FDG PET in Hodgkin's lymphoma: evaluation on 304 patients. Eur J Nucl Med Mol Imaging. 2012;39:4-12.

30. Floeth FW, Sabel M, Stoffels G, Pauleit D, Hamacher K, Steiger HJ, et al. Prognostic value of 18F-fluoroethyl-L-tyrosine PET and MRI in small nonspecific incidental brain lesions. J Nucl Med. 2008;49:730-7.

31. Barbet J, Chatal JF, Gauche F, Martino J. Which radionuclides will nuclear oncology need tomorrow? Eur J Nucl Med Mol Imaging. 2006;33:627-30. 